\title{
Magnetic and geometric control of spin textures in the itinerant kagome magnet $\mathrm{Fe}_{3} \mathrm{Sn}_{2}$
}

\author{
Markus Altthaler, ${ }^{1,2,3,{ }^{*}}$ Erik Lysne, ${ }^{2,3, *}$ Erik Roede $\odot,{ }^{2}$ Lilian Prodan,,${ }^{1}$ Vladimir Tsurkan,,${ }^{1,4}$ Mohamed A. Kassem $\odot,{ }^{5}$ \\ Hiroyuki Nakamura, ${ }^{6}$ Stephan Krohns $\odot,{ }^{1}$ István Kézsmárki, ${ }^{1, \dagger}$ and Dennis Meier ${ }^{2,3, \sharp}$ \\ ${ }^{1}$ Experimental Physics V, University of Augsburg, 86135 Augsburg, Germany \\ ${ }^{2}$ Department of Materials Science and Engineering, Norwegian University of Science and Technology (NTNU), 7043 Trondheim, Norway \\ ${ }^{3}$ Center for Quantum Spintronics, Department of Physics, Norwegian University of Science and Technology (NTNU), \\ 7491 Trondheim, Norway \\ ${ }^{4}$ Laboratory of Physics of Semiconductor Compounds, Institute for Applied Physics, MD-2028 Chisinau, Moldova \\ ${ }^{5}$ Department of Physics, Assiut University, 171516 Assiut, Egypt \\ ${ }^{6}$ Department of Materials Science and Engineering, Kyoto University, Kyoto 606-8501, Japan
}

(Received 16 June 2021; revised 17 September 2021; accepted 22 October 2021; published 17 December 2021)

\begin{abstract}
Magnetic materials with competing magnetocrystalline anisotropy and dipolar energies can develop a wide range of domain patterns, including classical stripe domains, domain branching, and topologically trivial and nontrivial (skyrmionic) bubbles. We image the magnetic domain pattern of $\mathrm{Fe}_{3} \mathrm{Sn}_{2}$ by magnetic force microscopy and study its evolution due to geometrical confinement, magnetic fields, and their combination. In $\mathrm{Fe}_{3} \mathrm{Sn}_{2}$ lamellae thinner than $3 \mu \mathrm{m}$, we observe stripe domains whose size scales with the square root of the lamella thickness, exhibiting classical Kittel scaling. Magnetic fields turn these stripes into a highly disordered bubble lattice. Complementary micromagnetic simulations quantitatively capture the magnetic field and thickness dependence of the magnetic patterns, reveal strong reconstructions of the patterns between the surface and the core of the lamellae, and identify the observed bubbles as skyrmionic bubbles. Our results imply that geometrical confinement together with competing magnetic interactions can provide a path to fine-tune and stabilize different types of topologically trivial and nontrivial spin structures in centrosymmetric magnets.
\end{abstract}

DOI: 10.1103/PhysRevResearch.3.043191

\section{INTRODUCTION}

Progress in information and communication technology is driven by the discovery of new materials and phenomena that enable components with improved functionality. In a recent development, topologically protected spin textures, such as magnetic skyrmions and merons, are explored as functional nanoscale objects that can be utilized to process and store information [1-3]. Initially linked to the Dzyaloshinskii-Moriya interaction (DMI) [4], skyrmions were mostly studied in non-centrosymmetric crystals, such as cubic chiral [5-7] and axially symmetric polar magnets [8-10]. It was demonstrated that the emergence of skyrmions often is accompanied by peculiar electromagnetic properties such as the topological Hall effect [11], a zoo of collective excitations [12-14], pinningfree motion at ultralow current densities, and polar dressing via the magnetoelectric effect [15].

The formation of skyrmions and topologically equivalent skyrmionic bubbles [16] is not restricted to non-

\footnotetext{
*These authors contributed equally to this work.

†istvan.kezsmarki@physik.uni-augsburg.de

‡dennis.meier@ntnu.no
}

Published by the American Physical Society under the terms of the Creative Commons Attribution 4.0 International license. Further distribution of this work must maintain attribution to the author(s) and the published article's title, journal citation, and DOI. centrosymmetric materials. In centrosymmetric magnets, such topologically nontrivial spin textures can be stabilized by frustrated exchange interactions [17] or originate from competing dipolar interactions and magnetic anisotropy [16,18-23]. In general, the spin texture of both skyrmions and skyrmionic bubbles is described by three parameters: the polarity ( $p= \pm 1$ ) corresponding to the direction of magnetization at the core, the helicity $(\gamma)$ determined by the offset to the polar phase $[\Phi(\phi)=m \phi+\gamma]$, and the vorticity

$$
m=\frac{1}{2 \pi} \int_{0}^{2 \pi} \mathrm{d} \Phi(\phi),
$$

which counts the number of revolutions of the polar angle [24]. Here, $\phi$ and $\Phi$ are the azimuthal angle in real space and the azimuthal angle of the local magnetization, respectively. In centrosymmetric magnets, bubbles with $m=0$ are topologically trivial, whereas $m=1$ indicates a topologically protected state, corresponding to skyrmions or skyrmionic bubbles. Depending on the relative magnitudes of the involved interactions, topologically trivial and nontrivial bubbles can also coexist, leading to complex mixed states [18,25].

For achieving such exotic magnetic states and, hence, novel functional responses, materials with comparable values of perpendicular uniaxial magnetocrystalline anisotropy (PMA) and shape anisotropy are particularly promising $[18,26]$. The delicate balance of the two anisotropy terms creates substantial frustration, which enables the formation of unconventional 
magnetic order, including modulated magnetic structures, Bloch points and lines, and skyrmionic bubbles with opposite helicity [18].

Here, we study the kagome crystal $\mathrm{Fe}_{3} \mathrm{Sn}_{2}$, i.e., an itinerant ferromagnet with competing uniaxial $\left(K_{u}\right)$ and shape anisotropies [27]. The latter is described by the quality factor $Q=2 K_{\mathrm{u}} / \mu_{0} M_{\mathrm{sat}}^{2}$, which has a value of about 0.15 . The material has a centrosymmetric rhombohedral structure formed of $\mathrm{Fe}-\mathrm{Sn}$ bilayers alternating with Sn layers along the crystallographic $c$ axis. The anomalous Hall effect [28-30], the exceptionally high Curie temperature, and massive Dirac fermions [30] in the vicinity of the Fermi energy make $\mathrm{Fe}_{3} \mathrm{Sn}_{2}$ highly attractive for technological applications [31,32]. Geometric confinement has been demonstrated to have a strong impact on the nucleation of bubbles. More precisely, bubbles with $m=0$ and $m=1$ (type II and type I, respectively) are permitted to coexist or topologically trivial bubbles are completely expelled [27]. Furthermore, by confining the geometry, Hou et al. [27] have demonstrated that the critical fields required to stabilize type I bubbles are significantly reduced, reaching realistic values for technological applications. Early studies on current-driven dynamics have also demonstrated that the helicity can be electrically tuned via spin transfer torque [33]. The presented experiments by Lorentz transmission electron microscopy (LTEM), however, were limited to electron-transparent samples with thicknesses $\lesssim 200 \mathrm{~nm}$. In order to expand the research and systemically study the impact of the demagnetization energy for thicknesses varying over larger length scales, we apply magnetic force microscopy (MFM). We find that micrometer-thick lamellashaped samples exhibit bulk-like behavior, developing the peculiar dendrite pattern reported for $\mathrm{Fe}_{3} \mathrm{Sn}_{2}$ bulk samples [26,34]. For smaller thickness $(\lesssim 1 \mu \mathrm{m})$, we observe the formation of stripe and bubble domains which decrease in size with decreasing lamella thickness, following Kittel's law [35]. In addition, we study how variable magnetic fields transform the magnetic stripe domains into skyrmionic bubbles while keeping the sample thickness fixed and, vice versa, how the magnetic structure evolves as the sample thickness varies under constant magnetic field. The MFM measurements are complemented by micromagnetic simulation of the three-dimensional (3D) magnetic order and its response to the applied manipulations, providing additional insight into the nanoscale physics of topological spin textures in magnetic materials with competing uniaxial and shape anisotropies.

\section{METHODS}

\section{A. Crystal growth}

$\mathrm{Fe}_{3} \mathrm{Sn}_{2}$ single crystals are grown by the chemical transport reactions method. As starting material for growth, the preliminary synthesized polycrystalline powder is used. It has been prepared by solid state reactions from the high-purity elements $\mathrm{Fe}(99.99 \%)$ and $\mathrm{Sn}(99.995 \%)$. Iodine is utilized as the transport agent in the single-crystal growth. The growth is performed at temperatures between 730 and $680{ }^{\circ} \mathrm{C}$. Plate-like samples of thickness $20-40 \mu \mathrm{m}$ along the $c$ axis and 3-5 mm within the $a b$ plane are obtained.
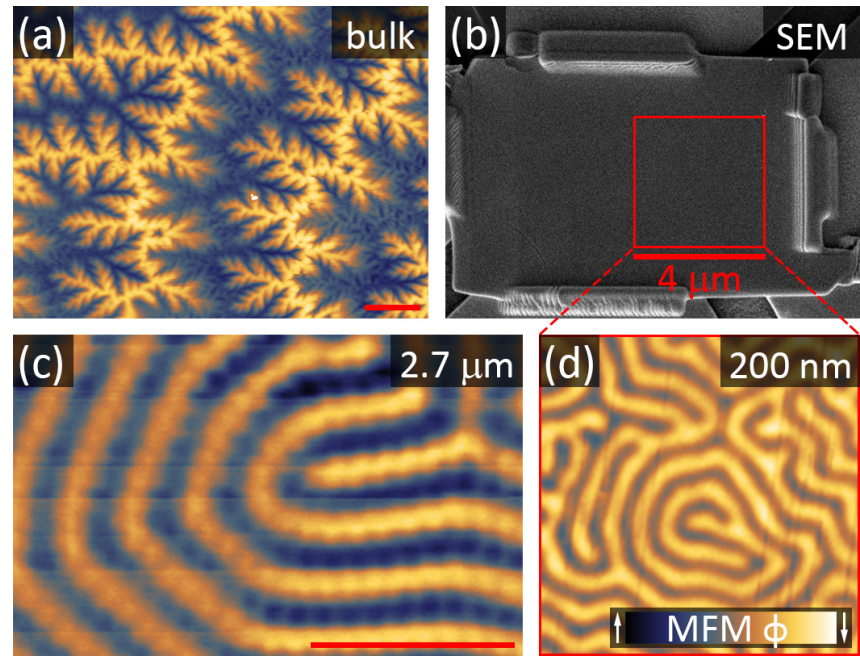

FIG. 1. (a) MFM image of a polished $a b$-plane surface of a $\mathrm{Fe}_{3} \mathrm{Sn}_{2}$ single crystal (dendrite pattern). (b) Representative SEM image showing a plan-view lamella cut from an $a b$-plane surface as shown in (a). (c) MFM image of a 2.7- $\mu$ m-thick lamella revealing a pattern of out-of-plane magnetized alternating ferromagnetic stripe domains. Each individual stripe exhibits an undulated pattern. (d) MFM scan of a 200-nm-thick lamella. All images are taken at room temperature, and the scale bars (red) are $4 \mu \mathrm{m}$.

\section{B. Lamella preparation}

$\mathrm{Fe}_{3} \mathrm{Sn}_{2}$ plan-view lamellae are prepared using a focused ion beam scanning electron microscope (FIB-SEM) at $30 \mathrm{kV}$ ion beam acceleration voltage. We employ a Thermo Scientific G4UX DualBeam and a Zeiss Crossbeam 550 system equipped with an EasyLift EX NanoManipulator and a Kleindiek MM3A-EM micromanipulator, respectively. A triangular prism-shaped volume is isolated by milling undercuts at $45^{\circ}$ to the $a b$ surface from a $\mathrm{Fe}_{3} \mathrm{Sn}_{2}$ single crystal. The specimen is then extracted using a micromanipulator and transferred to a copper half grid. By milling parallel to the original crystal surface, the samples are thinned to the desired thickness. The lamellae are then lifted off again and transferred to a substrate covered with $200 \mathrm{~nm}$ of gold. In order to attach the lamella, the edges are welded to the surface using carbon or platinum as seen in the SEM image in Fig. 1(b). Lamellae with controlled thicknesses between $200 \mathrm{~nm}$ and several micrometers are prepared and fixed flat on a substrate.

Following the same procedure, a prism-shaped volume is prepared for the wedged lamella. After polishing the original surface, the sample is transferred to a silicon substrate with the surface facing down. Mounted on a $45^{\circ}$ stub, the prism is then cut from the apex at a low angle versus the base plane resulting in a wedged lamella with a thickness varying from 400 to $900 \mathrm{~nm}$ along the long axis.

\section{Magnetic imaging}

To investigate the magnetic domain textures of $\mathrm{Fe}_{3} \mathrm{Sn}_{2}$, we perform MFM on an Oxford Instruments Cypher ES environmental atomic force microscope (AFM) at room temperature, mapping the phase shift of the readout signal. For 
low-temperature and field-dependent studies, an attocube attoAFM I, equipped with a superconducting magnet is used, recording the frequency shift with a phase-sensitive feedback loop. In both setups, the obtained image contrast is proportional to the projection of the gradient of the stray magnetic field to the tip magnetization [36]. In this paper, we use Nanosensors PPP-MFMR probes magnetized prior to the scans.

The periodicity of the magnetic patterns in the MFM images is quantified using a MATLAB script. At each point of the individual MFM images the local periodicity is determined by fast Fourier transformation (FFT) over an area that is sufficiently large not to affect the obtained periodicity by a spatial cutoff. For wedged samples, the local thickness is derived from the corresponding topography image, and the periodicity is averaged for equal specimen thickness.

\section{Micromagnetic simulations}

Micromagnetic simulations are performed using MUMAX3 [37]. The energy terms included in the model represent the Heisenberg exchange, first-order uniaxial anisotropy, Zeeman, and demagnetization energy terms,

$$
\begin{aligned}
\varepsilon=\int_{V_{\mathrm{s}}} & {\left[A_{\mathrm{ex}}(\nabla \mathbf{m})^{2}-K_{\mathrm{u}} m_{z}^{2}\right.} \\
& \left.\quad+M_{\mathrm{sat}} \mathbf{B}_{\mathrm{ext}} \cdot \mathbf{m}-\frac{1}{2} M_{\mathrm{sat}} \mathbf{B}_{\mathrm{dem}} \cdot \mathbf{m}\right] d \mathbf{r} .
\end{aligned}
$$

Here, $\mathbf{m}(x, y, z) \equiv \mathbf{M}(x, y, z) / M_{\mathrm{sat}}$ is the reduced magnetization. The orientation of the magnetization $\mathbf{M}(x, y, z)$ within the sample volume $V_{\mathrm{s}}$ is represented as a continuous unit vector field. $M_{\text {sat }}$ is the saturation magnetization, $A_{\text {ex }}$ is the exchange constant, $K_{\mathrm{u}}$ is the first-order uniaxial anisotropy constant, $\mathbf{B}_{\text {ext }}$ is the external magnetic field, and $\mathbf{B}_{\text {dem }}$ is the demagnetization field. Based on former studies at room temperature [27,31-33], the magnetic easy axis is chosen to point along the $z$ axis. In order to avoid edge-induced effects, periodic boundary conditions are applied along the in-plane directions $(x$ and $y)$. Open boundary conditions are used along the out-of-plane direction $(z|| c)$, reflecting the constraints of $a b$-plane cuts of varying thickness. The mesh is constructed of $8 \times 8 \times 8$-nm cells. The geometry is set to a square cuboid with length and width of $4096 \mathrm{~nm}$. We investigate the thickness dependence of the magnetic patterns for a series of cuboids with different thicknesses. The magnetization is relaxed from an initial uniform out-of-plane magnetized state, where nucleation sites are included in the form of cylinders magnetized in the opposite direction to the surrounding matrix. The latter allows for stabilizing the mazelike ground state observed experimentally against uniform saturation.

\section{RESULTS}

MFM scans of the polished $a b$-plane surface of a $\mathrm{Fe}_{3} \mathrm{Sn}_{2}$ crystal reveal a peculiar pattern of alternating bright and dark phase contrast, showing branched ferromagnetic domains [Fig. 1(a)]. Such domain branching is characteristic for $\mathrm{Fe}_{3} \mathrm{Sn}_{2}$ [34] and materials with PMA in general [26]. The order of branching is expected to scale with the width of the underlying domains, vanishing with decreasing sample (a)
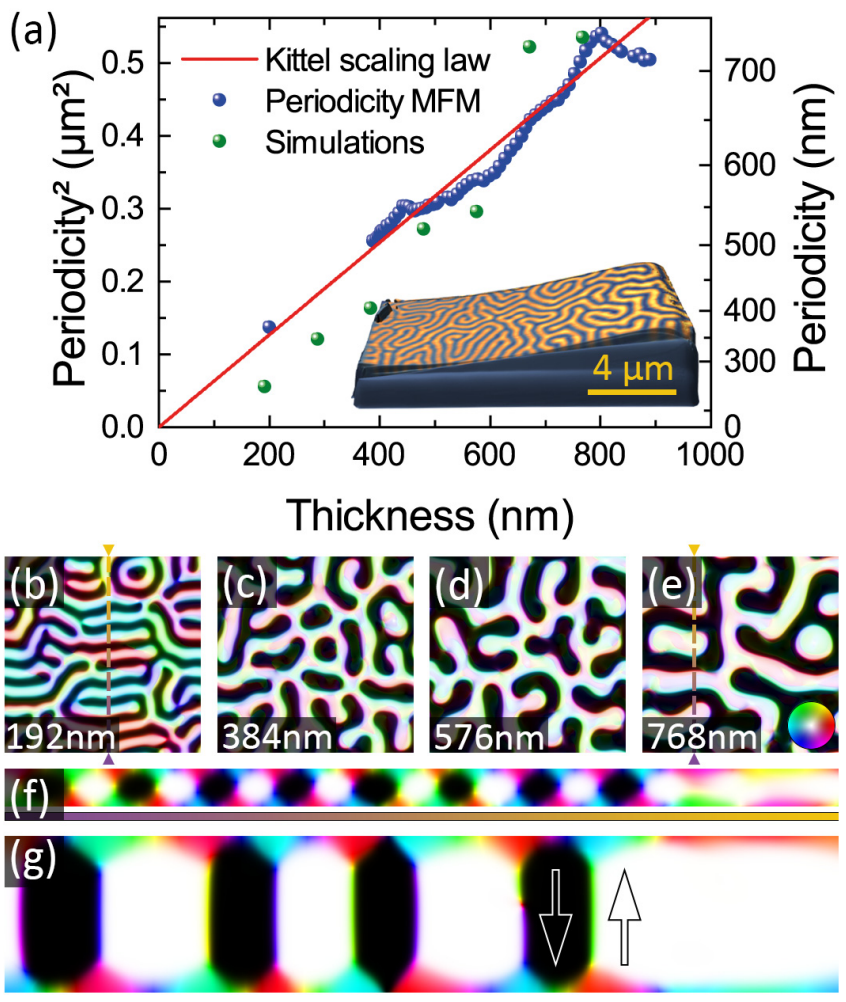

FIG. 2. (a) The inset displays the 3D visualization of the topography of the wedged lamella, at room temperature, colored with the magnetic texture. Bright and dark contrast corresponds to domains with out-of-plane magnetization pointing up and down, respectively. The local periodicity (blue data points) is obtained via fast Fourier transformation (FFT)-based analysis of the MFM signal and plotted vs the sample thickness. (b)-(e) Selection of simulated structures with thicknesses from 192 to $768 \mathrm{~nm}$, representing a $4 \times 4 \mu \mathrm{m}^{2}$ area with periodic boundary conditions. The green data points show the periodicity derived from simulations scaled by a factor of 1.12 to match the scaling law of the experimental data. (f) and (g) Representative cross sections ( $a c$ plane) for the thin and thick ends of the wedge lamella taken along the lines marked in (b) and (e). In all simulations, brightness corresponds to the out-of-plane component ( $c$ axis) of the magnetization, whereas colors encode the in-plane components ( $a b$ plane) in accordance with the color wheel in (e). Top-view images show a depth average of all layers, whereas cross sections represent an individual layer.

thickness [26]. Experimentally, we observe that the domain branching in $\mathrm{Fe}_{3} \mathrm{Sn}_{2}$ disappears at a sample thickness of about $2.7 \mu \mathrm{m}$. The corresponding MFM image, Fig. 1(c), reveals predominantly stripe domains with undulated domain walls characteristic for the onset of domain branching. In contrast, a mazelike pattern of stripe domains with straight domain walls is observed in thinner lamellae as presented in Fig. 1(d), consistent with literature [31]. The MFM data confirm that the applied FIB-based extraction procedure does not suppress or hinder the magnetic domain formation, confirming that the applied approach is suitable for preparation of high-quality $\mathrm{Fe}_{3} \mathrm{Sn}_{2}$ lamellae.

Figure 2 presents the effect of geometrical confinement. The possibility to utilize FIB milling to shape lamellae enables many opportunities to alter the geometry systematically and 
with nanometer precision. We seize this option to geometrically confine the material along the crystallographic $c$ axis. For this purpose, a wedged lamella with thickness ranging from 400 to $900 \mathrm{~nm}$ is prepared, allowing us to probe the continuous variation of the magnetic pattern as a function of the applied confinement in the $c$ direction. The MFM image recorded on the wedged lamella is displayed as an inset to Fig. 2(a). Qualitatively, the ferromagnetic stripe pattern resembles the magnetic domain state observed in the lamella in Fig. 1(d). Along the thickness gradient a clear widening of the stripes can be observed towards the thicker end of the lamella. Perpendicular to the gradient, no significant variation of the stripe width is found, pointing to a direct connection between the local thickness of the lamella and the width of the stripe domains. Our quantitative analysis shows a square-root dependence of the domain width on the lamella thickness, i.e., the stripe width follows classical Kittel scaling [35].

The variation of the stripe width versus sample thickness, revealed within the wedged lamella, provides a basis to refine the magnetic interaction parameters in $\mathrm{Fe}_{3} \mathrm{Sn}_{2}$ for more accurate micromagnetic simulations. Figures 2(b)-(e) show the results of our simulations for square cuboid lamellae with various thicknesses $(192,384,576$, and $768 \mathrm{~nm})$. With the parameter values $M_{\mathrm{sat}}=566 \mathrm{kA} / \mathrm{m}, A_{\mathrm{ex}}=14.0 \mathrm{pJ} / \mathrm{m}$, and $K_{\mathrm{u}}=30.0 \mathrm{~kJ} / \mathrm{m}^{3}$, the simulations quantitatively reproduce the thickness dependence of the stripe width [cf. Fig. 2(a)], as observed in the surface region of the lamella. Importantly, the refined micromagnetic simulations provide an in-depth view on the 3D magnetization pattern. Figures $2(\mathrm{f})$ and $2(\mathrm{~g})$ show cross-section images of the magnetization representative for the thin and thick ends of the wedged lamella, respectively. In both cases, alternating ferromagnetic $180^{\circ}$ domains, coaligned with the uniaxial anisotropy, constitute the core of the cuboids. In the surface region, Néel caps occur, explaining the gradual transition between domains in the MFM response. While the rotation of the magnetization is rather smooth in the surface region, the walls between up and down domains become considerably sharper in the internal region. This effect is strongest for the largest lamella thickness in Fig. 2(g).

After clarifying the thickness dependence of the magnetic texture, we investigate its magnetic-field-driven evolution. Figure 3 shows the field dependence of the magnetization for a macroscopic single-crystalline sample at $300 \mathrm{~K}$ with the magnetic field applied out of plane, i.e., parallel to the $c$ axis. In zero magnetic field, the sample has no remanent magnetization in agreement with the stripe-domain pattern observed by MFM and in the micromagnetic simulations. Following a linear increase, the magnetization saturates at $750 \mathrm{mT}$ to the value of $2 \mu_{\mathbf{B}}$ per Fe atom. This value is in good agreement with earlier reports [38,39] and converts to $M_{\text {sat }}=571 \mathrm{kA} / \mathrm{m}$, which agrees within $1 \%$ with the value refined in the micromagnetic simulations. The detailed field evolution of the domain pattern is documented by a series of MFM images recorded on a 450-nm-thick $\mathrm{Fe}_{3} \mathrm{Sn}_{2}$ lamella at $300 \mathrm{~K}$. MFM images recorded over the same area in 200-mT steps are shown both for increasing the field up to saturation and for decreasing it back to zero. Additional MFM data recorded at $200 \mathrm{~K}$ are presented in Fig. 5 of Appendix A,

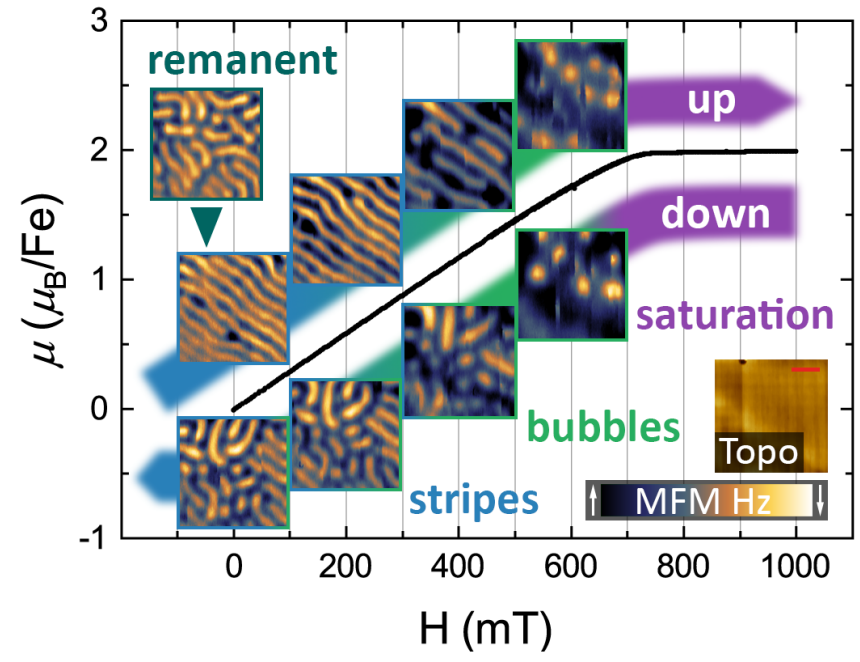

FIG. 3. The magnetization data for a macroscopic $\mathrm{Fe}_{3} \mathrm{Sn}_{2}$ sample (presented by the black data points), taken at $T=300 \mathrm{~K}$, indicate that saturation magnetization is reached at about $750 \mathrm{mT}$ and a hysteresis-free cycle. Magnetic-field-dependent MFM scans recorded on a 450-nm-thick lamella are presented as insets. The bottom right inset shows the underlying topography (Topo) of the $4 \times 4 \mu \mathrm{m}^{2}$ area evaluated $(T=300 \mathrm{~K})$, with a $1-\mu \mathrm{m}$ scale bar. The top left inset shows the virgin, i.e., a random remanent, state, prior to ordering the stripes by the in-plane field. The direction of the field ramp and the predominant domain type are indicated by the underlying arrows and their color, respectively. The insets (frames colored analog) elucidate the domain evolution.

showing a qualitatively equivalent response of the domain structure to the applied magnetic field. In both cases, we observe that with increasing magnetic field, the area magnetized opposite to the field shrinks. Subsequently, the stripe domains gradually transform to bubble domains in between 300 and $600 \mathrm{mT}$ before being expunged at the transition to the uniform ferromagnetic phase at approximately $700 \mathrm{mT}$. With decreasing field, bubble domains form spontaneously just below $700 \mathrm{mT}$ and eventually elongate. In $600 \mathrm{mT}$, i.e., just below the saturation field, a pure bubble phase is realized. Note that these can be either type I or type II bubbles as the MFM experiment cannot distinguish between them. Below $500 \mathrm{mT}$ the fragmented stripes gradually grow and merge at low fields. Thus, the behavior observed in the lamella is qualitatively similar to the domain evolution observed in classical ferromagnets with PMA, such as $\mathrm{CoCr}$ and $\mathrm{TbGdFeCo}$, under application of a magnetic field [26]. The emergence of completely different domain wall positions at zero field before and after ramping the magnetic field further reflects that no substantial pinning occurs in the $\mathrm{Fe}_{3} \mathrm{Sn}_{2}$ lamella. Thus, pronounced effects from defects, e.g., Ga implantation from the FIB preparation, can be excluded.

Next, we investigate the combined effect of thickness variation and magnetic field on the domain patterns in $\mathrm{Fe}_{3} \mathrm{Sn}_{2}$ by studying the domain structure in a wedged lamella as a function of magnetic field. The experimental results and complementary micromagnetic simulations, displayed in Fig. 4, show the magnetic textures in zero field [Fig. 4(a)] and $450 \mathrm{mT}$ [Fig. 4(b)]. In zero field, stripes form a mazelike 


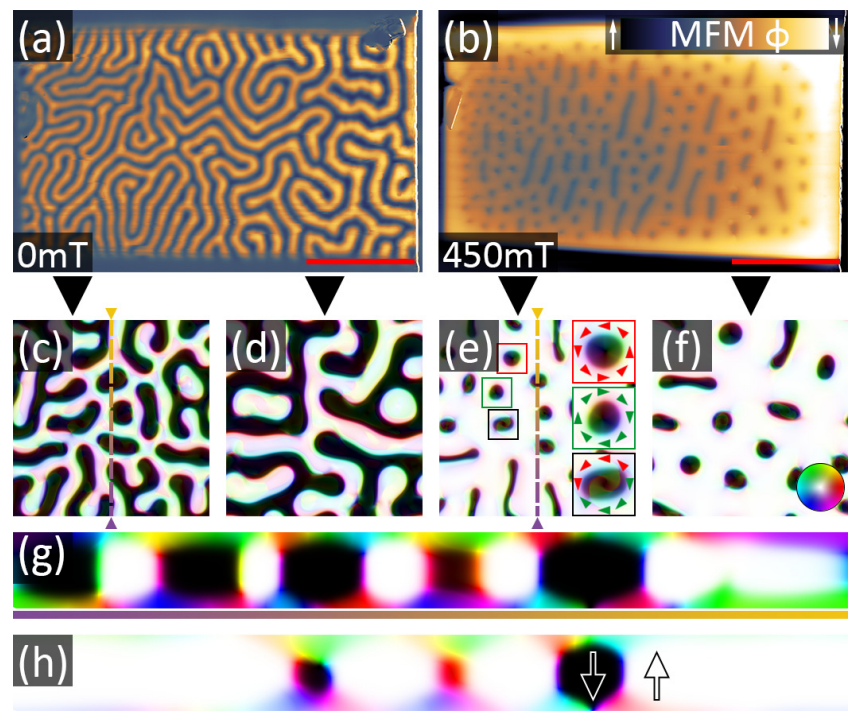

FIG. 4. (a) and (b) show MFM images of the wedge-shaped lamella in zero field and $450 \mathrm{mT}$ (applied along the out-of-plane direction). Both data sets are taken at room temperature, and the scale bars (red) are $4 \mu \mathrm{m}$. (c) and (d) calculated magnetic texture in a region of $4 \times 4 \mu \mathrm{m}^{2}$, considering a sample thickness of 384 and $768 \mathrm{~nm}$, respectively, which represent the thin and thick ends of the lamella in zero field. (e) and (f) calculated magnetic textures in an external field of $450 \mathrm{mT}$, showing the field evolution of (c) and (d), respectively. The insets to (e) show the coexistence of skyrmionic bubbles of opposite helicity (red, green), as well as trivial bubbles (black). (g) and (h) show cross sections (ac plane) illustrating the field evolution at the lines marked in (c) and (e). In all simulations, brightness corresponds to the out-of-plane component ( $c$ axis) of the magnetization, whereas colors encode the in-plane components ( $a b$ plane) in accordance with the color wheel in (f). Top-view images show a depth average of all layers, whereas cross sections represent an individual layer.

pattern, where the stripe width scales with the thickness according to Kittel's law, as presented in Fig. 2(a). In 450 mT, this pattern transforms to a state where shorter stripes coexist with bubbles. The nucleation of bubbles seems to start at the edges of the lamella, whereas the remaining stripes are concentrated within the internal part of the lamella. The phase coexistence of stripes and bubbles in $450 \mathrm{mT}$ is consistent with the magnetic-field-dependent data recorded at 300 and $200 \mathrm{~K}$ (see Figs. 3 and 5). Interestingly, we observe that not only the remaining fragmented stripe domains shrink in size with decreasing thickness, but also the diameter of the newly formed bubbles scales accordingly, as presented in Fig. 6 of Appendix B, following Kittel's scaling law.

Micromagnetic simulations representing the domain structure in the thin and the thick ends of the lamella, respectively, are shown in Figs. 4(c) and 4(d) (same as in Figs. 2(c) and 2(e)). The simulations reproduce the domain pattern observed in zero field, including the thickness dependence of the stripe width as already discussed in detail for Fig. 2. In $450 \mathrm{mT}$, similar to the experimental data, a mixed state of residual stripe domains and bubble domains is realized in the simulation, as shown in Figs. 4(e) and 4(f). The insets to Fig. 4(e) emphasize the coexistence of skyrmionic and topologically trivial bubbles, which cannot be concluded from the MFM scans alone. Furthermore, the simulated 3D magnetization reveals bubbles of opposite helicity. Cross-section views of the thin end of the sample in zero field and $450 \mathrm{mT}$, Figs. 4(g) and 4(h), show the magnetic texture in the core of the sample. In zero field, the pattern is similar to that found in the thick sample displayed in Fig. 2(g). In $450 \mathrm{mT}$, the area magnetized parallel to the field is extended, and the remaining bubble domains of opposite magnetization are confined by sharp domain walls in the core and clipped at the surface by the in-plane closure domain pattern, i.e., the Néel caps. As a consequence, the diameter of the bubble domains varies substantially between core and surface.

\section{SUMMARY}

In this paper, we studied the response of the micromagnetic spin texture in the frustrated kagome magnet $\mathrm{Fe}_{3} \mathrm{Sn}_{2}$ under geometrical confinement by reducing the thickness along the crystallographic $c$ direction and in a magnetic field. It has been shown that the electronic structure of this magnetic Weyl semimetal critically depends on the orientation of the magnetization [40]. Therefore detailed magnetic imaging studies revealing the characteristics of magnetic domains in constrained, as well as unconstrained, sample geometries are important, being relevant not only in terms of pure magnetic phenomena in $\mathrm{Fe}_{3} \mathrm{Sn}_{2}$ but also for the interpretation of magneto-transport and magneto-optical experiments, and electronic structure calculations. Bulk samples reveal a branched [26] pattern of alternating ferromagnetic domains, which is characteristic for materials with PMA. In contrast, lamellae with thicknesses on the order of a few hundred nanometers develop stripe patterns of alternating ferromagnetic $180^{\circ}$ domains. The thickness limit for the onset of domain branching is about $2.7 \mu \mathrm{m}$. Below this limit, the size of the stripe domains follows classical Kittel scaling. The experimental results are corroborated by micromagnetic simulations, implying that the competition between dipolar interactions and uniaxial anisotropy is the driving force behind the formation and scaling of the domains. Furthermore, the micromagnetic simulations reveal Néel caps, i.e., the formation of closure domains at the surface. Analogous to the response to geometrical confinement, classical domain behavior is also oberved under external magnetic fields. We find that stripe domains contract and break up into bubbles before a field-polarized single-domain state is reached. Intermediate $Q$ materials, such as $\mathrm{Fe}_{3} \mathrm{Sn}_{2}$, exhibit classical domain responses, thus enabling domain engineering following established strategies: introduction of geometrical confinement and application of an external magnetic field. Importantly, this behavior also applies to both topologically trivial and topologically nontrivial bubble domains. This yields the opportunity to tailor the host material to develop specific skyrmionic bubbles and mixed states with optimized domain size and densities for potential future applications. 


\section{ACKNOWLEDGMENTS}

This work was supported by the DFG via the Transregional Research Collaboration TRR 80 From Electronic Correlations to Functionality (Augsburg/Munich/Stuttgart) and via the DFG Priority Program SPP2137, Skyrmionics, under Grant No. KE 2370/1-1 and by the project ANCD 20.80009.5007.19 (Moldova). E.L., E.R., and D.M. thank the Research Council of Norway for funding (Project No. 263228) and support through the Norwegian Micro- and Nano-Fabrication Facility, NorFab (Project No. 295864). M.A., E.L., and D.M. acknowledge support by the Research Council of Norway through its Centres of Excellence funding scheme, Project No. 262633, "QuSpin." D.M. thanks NTNU for support via the Onsager Fellowship Program and the Outstanding Academic Fellows Program.
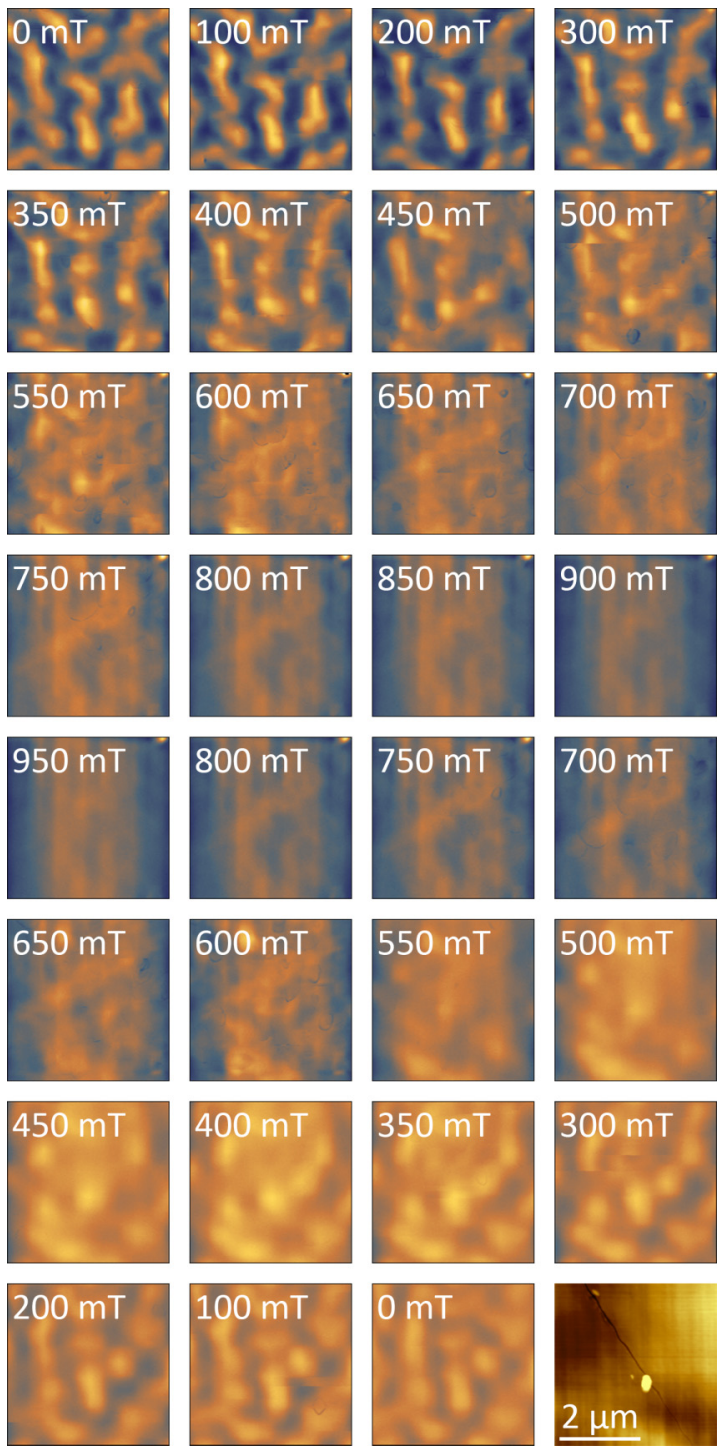

FIG. 5. MFM images showing the field evolution of domains at $200 \mathrm{~K}$. The bottom right image displays the corresponding topography of the $4 \times 4 \mu \mathrm{m}^{2}$ area.

\section{APPENDIX A: LOW-TEMPERATURE FIELD EVOLUTION}

In addition to the MFM data presented in Fig. 3 of the main text, magnetic-field-dependent measurements were conducted at $200 \mathrm{~K}$. Figure 5 shows the respective MFM image series taken in 50-mT steps (lower field values are in steps of $100 \mathrm{mT}$ ) up to $950 \mathrm{mT}$. A reversible transition from stripe to bubble domains is observed before the uniform ferromagnetic state sets in, analogous to the behavior at $300 \mathrm{~K}$.

\section{APPENDIX B: THICKNESS-DEPENDENT SCALING OF BUBBLE DOMAINS}

Figure 2(a) in the main text addresses classical Kittel scaling of stripe domain patterns observed in zero field. To show that the bubble domains follow the same trend as the stripe domains, we evaluate the size of bubble domains for three thicknesses as presented in Fig. 6(a). (Note that due to the asymmetric field response of up and down domains, the bubble-dominated pattern is no longer periodic, which prohibits a rigorous analysis via the same method we applied for the stripe domains.) Figure 6(b) presents the respective five bubbles analyzed for each approximate thickness. The diameter is derived from the FWHM of three cross sections per bubble and subsequent averaging.
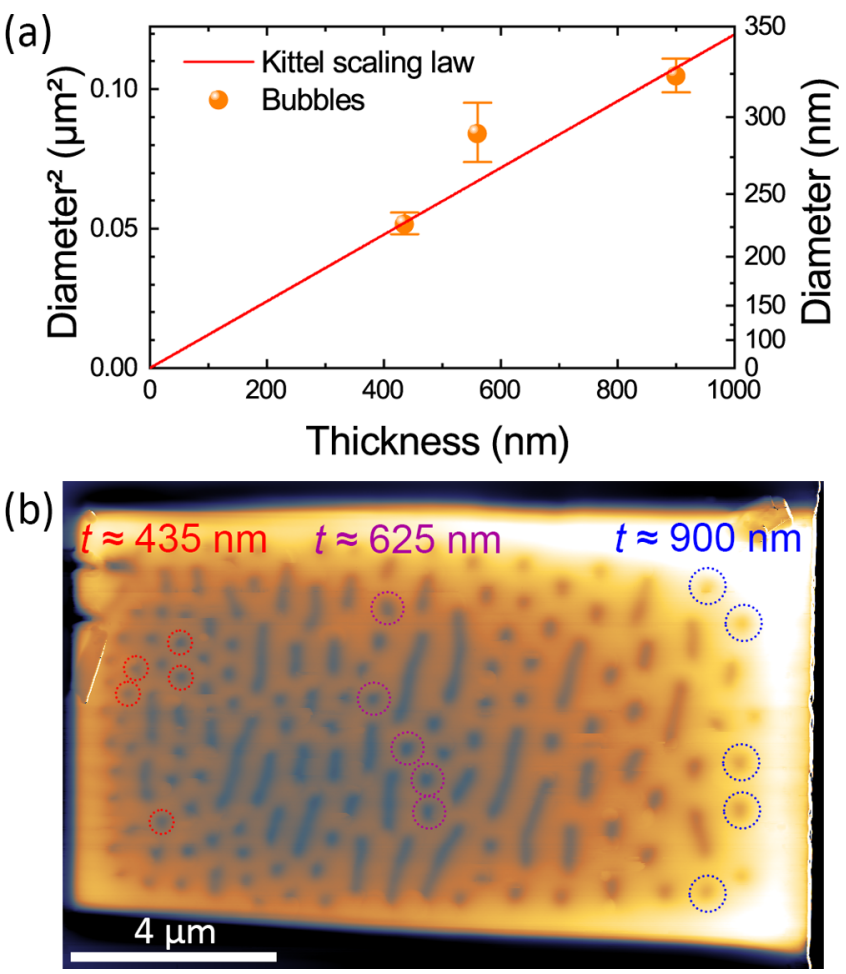

FIG. 6. (a) Scaling of representative bubbles, emerging in the wedge-shaped lamella due to the application of a $450 \mathrm{mT}$ field along the $c$ axis. (b) Overview of selected bubbles and the corresponding approximate thickness of the lamella. 
[1] T. H. R. Skyrme, A unified field theory of mesons and baryons, Nucl. Phys. 31, 556 (1962).

[2] R. Tomasello, E. Martinez, R. Zivieri, L. Torres, M. Carpentieri, and G. Finocchio, A strategy for the design of skyrmion racetrack memories, Sci. Rep. 4, 6784 (2014).

[3] X. Zhang, G. P. Zhao, H. Fangohr, J. P. Liu, W. X. Xia, J. Xia, and F. J. Morvan, Skyrmion-skyrmion and skyrmion-edge repulsions in skyrmion-based racetrack memory, Sci. Rep. 5, 7643 (2015).

[4] I. Dzyaloshinsky, A thermodynamic theory of "weak" ferromagnetism of antiferromagnetics, J. Phys. Chem. Solids 4, 241 (1958).

[5] S. Mühlbauer, B. Binz, F. Jonietz, C. Pfleiderer, A. Rosch, A. Neubauer, R. Georgii, and P. Böni, Skyrmion lattice in a chiral magnet, Science 323, 915 (2009).

[6] W. Münzer, A. Neubauer, T. Adams, S. Mühlbauer, C. Franz, F. Jonietz, R. Georgii, P. Böni, B. Pedersen, M. Schmidt, A. Rosch, and C. Pfleiderer, Skyrmion lattice in the doped semiconductor $\mathrm{Fe}_{1-x} \mathrm{Co}_{x} \mathrm{Si}$, Phys. Rev. B 81, 041203(R) (2010).

[7] X. Z. Yu, N. Kanazawa, Y. Onose, K. Kimoto, W. Z. Zhang, S. Ishiwata, Y. Matsui, and Y. Tokura, Near room-temperature formation of a skyrmion crystal in thin-films of the helimagnet FeGe, Nat. Mater. 10, 106 (2011).

[8] I. Kézsmárki, S. Bordács, P. Milde, E. Neuber, L. M. Eng, J. S. White, H. M. Rønnow, C. D. Dewhurst, M. Mochizuki, K. Yanai, H. Nakamura, D. Ehlers, V. Tsurkan, and A. Loidl, Néeltype skyrmion lattice with confined orientation in the polar magnetic semiconductor $\mathrm{GaV}_{4} \mathrm{~S}_{8}$, Nat. Mater. 14, 1116 (2015).

[9] Á. Butykai, S. Bordács, I. Kézsmárki, V. Tsurkan, A. Loidl, J. Döring, E. Neuber, P. Milde, S. C. Kehr, and L. M. Eng, Characteristics of ferroelectric-ferroelastic domains in Néeltype skyrmion host $\mathrm{GaV}_{4} \mathrm{~S}_{8}$, Sci. Rep. 7, 44663 (2017).

[10] S. Bordács, A. Butykai, B. G. Szigeti, J. S. White, R. Cubitt, A. O. Leonov, S. Widmann, D. Ehlers, H.-A. K. von Nidda, V. Tsurkan, A. Loidl, and I. Kézsmárki, Equilibrium skyrmion lattice ground state in a polar easy-plane magnet, Sci. Rep. 7, 7584 (2017).

[11] A. Neubauer, C. Pfleiderer, B. Binz, A. Rosch, R. Ritz, P. G. Niklowitz, and P. Böni, Topological Hall Effect in the $a$ Phase of MnSi, Phys. Rev. Lett. 102, 186602 (2009).

[12] M. Garst, J. Waizner, and D. Grundler, Collective spin excitations of helices and magnetic skyrmions: review and perspectives of magnonics in non-centrosymmetric magnets, J. Phys. D: Appl. Phys. 50, 293002 (2017).

[13] T. Schwarze, J. Waizner, M. Garst, A. Bauer, I. Stasinopoulos, H. Berger, C. Pfleiderer, and D. Grundler, Universal helimagnon and skyrmion excitations in metallic, semiconducting and insulating chiral magnets, Nat. Mater. 14, 478 (2015).

[14] D. Ehlers, I. Stasinopoulos, V. Tsurkan, H.-A. Krug von Nidda, T. Fehér, A. Leonov, I. Kézsmárki, D. Grundler, and A. Loidl, Skyrmion dynamics under uniaxial anisotropy, Phys. Rev. B 94, 014406 (2016).

[15] E. Ruff, S. Widmann, P. Lunkenheimer, V. Tsurkan, S. Bordács, I. Kézsmárki, and A. Loidl, Multiferroicity and skyrmions carrying electric polarization in $\mathrm{GaV}_{4} \mathrm{~S} 8$, Sci. Adv. 1, e1500916 (2015).

[16] X. Yu, M. Mostovoy, Y. Tokunaga, W. Zhang, K. Kimoto, Y. Matsui, Y. Kaneko, N. Nagaosa, and Y. Tokura, Magnetic stripes and skyrmions with helicity reversals, Proc. Natl. Acad. Sci. USA 109, 8856 (2011).
[17] T. Okubo, S. Chung, and H. Kawamura, Multiple- $q$ States and the Skyrmion Lattice of the Triangular-Lattice Heisenberg Antiferromagnet under Magnetic Fields, Phys. Rev. Lett. 108, 017206 (2012).

[18] X. Yu, Y. Tokunaga, Y. Taguchi, and Y. Tokura, Variation of topology in magnetic bubbles in a colossal magnetoresistive manganite, Adv. Mater. 29, 1603958 (2017).

[19] W. Wang, Y. Zhang, G. Xu, L. Peng, B. Ding, Y. Wang, Z. Hou, X. Zhang, X. Li, E. Liu, S. Wang, J. Cai, F. Wang, J. Li, F. Hu, G. Wu, B. Shen, and X.-X. Zhang, A centrosymmetric hexagonal magnet with superstable biskyrmion magnetic nanodomains in a wide temperature range of 100-340 K, Adv. Mater. 28, 6887 (2016).

[20] J. Tang, L. Kong, Y. Wu, W. Wang, Y. Chen, Y. Wang, J. Li, Y. Soh, Y. Xiong, M. Tian, and H. Du, Target Bubbles in $\mathrm{Fe}_{3} \mathrm{Sn}_{2}$ Nanodisks at Zero Magnetic Field, ACS Nano 14, 10986 (2020).

[21] S. A. Montoya, S. Couture, J. J. Chess, J. C. T. Lee, N. Kent, D. Henze, S. K. Sinha, M.-Y. Im, S. D. Kevan, P. Fischer, B. J. McMorran, V. Lomakin, S. Roy, and E. E. Fullerton, Tailoring magnetic energies to form dipole skyrmions and skyrmion lattices, Phys. Rev. B 95, 024415 (2017).

[22] B. Ding, J. Cui, G. Xu, Z. Hou, H. Li, E. Liu, G. Wu, Y. Yao, and W. Wang, Manipulating Spin Chirality of Magnetic Skyrmion Bubbles by In-Plane Reversed Magnetic Fields in $\left(\mathrm{Mn}_{1-x} \mathrm{Ni}_{x}\right)_{65} \mathrm{Ga}_{35}(x=0.45)$ Magnet, Phys. Rev. Appl. 12, 054060 (2019).

[23] B. Ding, Z. Li, G. Xu, H. Li, Z. Hou, E. Liu, X. Xi, F. Xu, Y. Yao, and W. Wang, Observation of Magnetic Skyrmion Bubbles in a van der Waals Ferromagnet $\mathrm{Fe}_{3} \mathrm{GeTe}_{2}$, Nano Lett. 20, 868 (2020).

[24] B. Göbel, I. Mertig, and O. A. Tretiakov, Beyond skyrmions: Review and perspectives of alternative magnetic quasiparticles, Phys. Rep. 895, 1 (2021).

[25] J. C. Loudon, A. C. Twitchett-Harrison, D. Cortés-Ortuño, M. T. Birch, L. A. Turnbull, A. Štefančič, F. Y. Ogrin, E. O. Burgos-Parra, N. Bukin, A. Laurenson, H. Popescu, M. Beg, O. Hovorka, H. Fangohr, P. A. Midgley, G. Balakrishnan, and P. D. Hatton, Do Images of Biskyrmions Show Type-II Bubbles?, Adv. Mater. 31, 1806598 (2019).

[26] A. Hubert, Magnetic Domains: The Analysis of Magnetic Microstructures (Springer, NewYork, 1998), pp. XXIII, 696.

[27] Z. Hou, Q. Zhang, G. Xu, S. Zhang, C. Gong, B. Ding, H. Li, F. Xu, Y. Yao, E. Liu, G. Wu, X.-X. Zhang, and W. Wang, Manipulating the topology of nanoscale skyrmion bubbles by spatially geometric confinement, ACS Nano 13, 922 (2019).

[28] T. Kida, L. A. Fenner, A. A. Dee, I. Terasaki, M. Hagiwara, and A. S. Wills, The giant anomalous Hall effect in the ferromagnet $\mathrm{Fe}_{3} \mathrm{Sn}_{2}$-A frustrated kagome metal, J. Phys.: Condens. Matter 23, 112205 (2011).

[29] Q. Wang, S. Sun, X. Zhang, F. Pang, and H. Lei, Anomalous Hall effect in a ferromagnetic $\mathrm{Fe}_{3} \mathrm{Sn}_{2}$ single crystal with a geometrically frustrated Fe bilayer kagome lattice, Phys. Rev. B 94, 075135 (2016).

[30] L. Ye, M. Kang, J. Liu, F. von Cube, C. R. Wicker, T. Suzuki, C. Jozwiak, A. Bostwick, E. Rotenberg, D. C. Bell, L. Fu, R. Comin, and J. G. Checkelsky, Massive Dirac fermions in a ferromagnetic kagome metal, Nature (London) 555, 638 (2018).

[31] Z. Hou, W. Ren, B. Ding, G. Xu, Y. Wang, B. Yang, Q. Zhang, Y. Zhang, E. Liu, F. Xu, W. Wang, G. Wu, X. Zhang, B. Shen, 
and Z. Zhang, Observation of various and spontaneous magnetic skyrmionic bubbles at room temperature in a frustrated kagome magnet with uniaxial magnetic anisotropy, Adv. Mater. 29, 1701144 (2017)

[32] Z. Hou, Q. Zhang, G. Xu, C. Gong, B. Ding, Y. Wang, H. Li, E. Liu, F. Xu, H. Zhang, Y. Yao, G. Wu, X.-X. Zhang, and W. Wang, Creation of single chain of nanoscale skyrmion bubbles with record-high temperature stability in a geometrically confined nanostripe, Nano Lett. 18, 1274 (2018).

[33] Z. Hou, Q. Zhang, X. Zhang, G. Xu, J. Xia, B. Ding, H. Li, S. Zhang, N. M. Batra, P. M. F. J. Costa, E. Liu, G. Wu, M. Ezawa, X. Liu, Y. Zhou, X. Zhang, and W. Wang, Currentinduced helicity reversal of a single skyrmionic bubble chain in a nanostructured frustrated magnet, Adv. Mater. 32, 1904815 (2020).

[34] K. Heritage, B. Bryant, L. A. Fenner, A. S. Wills, G. Aeppli, and Y.-A. Soh, Images of a first-order spin-reorientation phase transition in a metallic kagome ferromagnet, Adv. Funct. Mater. 30, 1909163 (2020).
[35] C. Kittel, Theory of the structure of ferromagnetic domains in films and small particles, Phys. Rev. 70, 965 (1946).

[36] O. Kazakova, R. Puttock, C. Barton, H. Corte-León, M. Jaafar, V. Neu, and A. Asenjo, Frontiers of magnetic force microscopy, J. Appl. Phys. 125, 060901 (2019).

[37] A. Vansteenkiste, J. Leliaert, M. Dvornik, M. Helsen, F. GarciaSanchez, and B. Van Waeyenberge, The design and verification of MuMax3, AIP Adv. 4, 107133 (2014).

[38] G. L. Caer, B. Malaman, and B. Roques, Mossbauer effect study of $\mathrm{Fe}_{3} \mathrm{Sn}_{2}$, J. Phys. F: Met. Phys. 8, 323 (1978).

[39] B. Malaman, D. Fruchart, and G. L. Caer, Magnetic properties of $\mathrm{Fe}_{3} \mathrm{Sn}_{2}$. II. Neutron diffraction study (and Mossbauer effect), J. Phys. F: Met. Phys. 8, 2389 (1978).

[40] J.-X. Yin, S. S. Zhang, H. Li, K. Jiang, G. Chang, B. Zhang, B. Lian, C. Xiang, I. Belopolski, H. Zheng, T. A. Cochran, S.-Y. Xu, G. Bian, K. Liu, T.-R. Chang, H. Lin, Z.-Y. Lu, Z. Wang, S. Jia, W. Wang et al., Giant and anisotropic many-body spin-orbit tunability in a strongly correlated kagome magnet, Nature (London) 562, 91 (2018). 